\title{
Is There an Interaction between Task Complexity and Practice Variability in Speech-Motor learning?
}

\author{
Ramesh Kaipa \\ Department of Communication Sciences and Disorders, Oklahoma State University, Stillwater, Okla., USA
}

\section{Key Words}

Practice variability $\cdot$ Task complexity $\cdot$ Speech-motor learning

\begin{abstract}
Background: Prior studies have investigated the influence of principles of motor learning (PMLs) on speech-motor learning. However, the interactive effect of different PMLs on speech-motor learning remains unknown. Purpose: This study is aimed at investigating the interaction of $2 \mathrm{PMLs}$, that is, practice variability and task complexity and their influence on speech-motor learning. Method: Forty healthy individuals (aged 18-30 years) were randomly and equally allocated to 2 groups where they had to either practice a simple (simple group) or a complex phrase (complex group). Two levels of practice variability (constant and variable) were considered in training participants in simple and complex groups. Participants practiced 50 practice trials of either complex or simple phrase during the first 2 days. At the end of training on each day, participants produced 10 trials of the phrase they practiced without feedback. On the third day, participants returned for a delayed retention test. The participant utterances on all the 3 days were recorded for later analysis. Results: Data analysis revealed that there was no major effect of practice condition, and there was no interaction of task complexity and practice condition. However,
\end{abstract}

there was an interaction between data collection points and complexity. Conclusion: The findings suggest that irrespective of the complexity of the to-be-learned speech task, there is no preponderance of variable over constant practice, which contradicts the findings of the non-speech-motor learning literature.

(c) 2016 S. Karger AG, Basel

\section{Introduction}

Principles of motor learning (PMLs) refer to guided principles that can facilitate motor skill learning when applied systematically [1-3]. PMLs can be classified into 2 types: (1) principles pertaining to the structure of practice and (2) principles pertaining to the nature of feedback [4]. Structure of practice refers to the act of rehearsing behavior repeatedly for the purpose of mastering it [5]. A practice regime can be structured based on variables such as practice amount, practice distribution, practice variability, practice schedule, attention source, and task complexity [6]. Nature of feedback refers to the information related to the sensation associated with the movement itself [7]. Efficient feedback can be provided based on frequency, type, and timing of feedback [6]. PMLs have largely emerged from studies involving non-speech-motor tasks (e.g., archery). Recent studies have been investigating the

\section{KARGER}

E-Mail karger@karger.com

www.karger.com/aon
(C) 2016 S. Karger AG, Basel

0972-7531/16/0233-0134\$39.50/0 
role of PMLs in speech-motor learning [8-14]. An aspect of PMLs to have gained considerable attention in speechmotor learning is practice variability $[8,15,16]$. Practice variability can range from practicing just one variant of a motor skill (constant practice) to practicing 2 or more variants of the same motor skill (variable practice). In speech-motor learning, variable practice is found to be beneficial over constant practice [8].

In spite of the established benefits of variable practice over constant practice, the interactive effect of practice variability with other PMLs has not been investigated in speech-motor learning. Prior studies in non-speech-motor learning reveal the interaction of practice variability with other PMLs such as practice schedule, practice amount, and feedback frequency [17]. In case of speechmotor learning, one of the PMLs that is likely to interact with practice variability is task complexity. Past studies in speech-motor learning have used a variety of speech stimuli for practice ranging from meaningful monosyllabic word (e.g., 'green') [18] to bisyllabic non words (e.g., 'Thak glers wur vasing veen arad moovly') $[14,19]$. However, these studies have failed to consider the possible interactive effect of task complexity and practice conditions on speech-motor learning. It is possible that the effects of practice variability on speech-motor learning could vary based on the interaction with other PMLs such as task complexity $[4,20]$. It is essential to understand how task complexity interacts with other PMLs so that speech therapists are able to design appropriate stimuli for speech therapy and engage in evidence-based practice. Hence, the purpose of this study was to investigate if there was an interactive effect of practice variability and task complexity on learning novel speech utterances. Two levels of practice variability (constant and variable practice) and 2 levels of task complexity (simple and complex) were considered.

\section{Methods}

\section{Participants}

Forty healthy individuals (4 males and 36 females) in the age range of 18-30 years participated in the study. Participants were recruited at the author's institution based on a non-probability convenience sampling and were not matched according to sex. The inclusion criteria were: (1) no reported history of sensory, motor, or cognitive abnormalities, (2) native speaker of standard US English dialect, and (3) completion of a high school diploma. Participants were randomly and equally assigned to one of 4 practice conditions mentioned below. This study received ethical approval from the Institutional Review Board at the author's university and all participants provided written consent.

Interaction of Task Complexity and

Practice in Speech-Motor Learning

\section{Practice Conditions}

Of the 40 participants, 20 were required to practice a complex speech phrase ('complex group'). The remaining 20 participants practiced a simple phrase ('simple group'). Two levels of practice variability (constant and variable practice) were considered in training participants in complex and simple groups. The interaction of 2 levels of task complexity and 2 levels of practice variability resulted in 4 practice conditions: (1) simple constant, (2) simple variable, (3) complex constant, and (4) complex variable.

\section{Procedure}

Practice Design for the Complex Group

Complex group participants practiced a complex meaningless target phrase 'Thak glers wur vasing veen arad moovly'. A nonmeaningful speech phrase was chosen to avoid linguistic familiarity and ceiling effect during practice sessions. This phrase was also used as a training stimulus in a previous speech-motor learning study [12] involving healthy individuals, and was thus considered to be an appropriate stimulus for this study. The target complex phrase consisted of 7 non-words, 4 of them being monosyllabic, and 3 being bisyllabic. Even though the target phrase was not meaningful, it followed the constraints of English phonotactics. Among the 20 participants in the complex group, 10 participants were involved in constant practice of the target complex phrase, and another 10 participants were involved in variable practice.

The experiment lasted for 3 consecutive days. The first 2 days comprised the acquisition phase, and the third day was the retention phase. During each day of the acquisition phase, the participants practiced 50 trials of the complex phrase. The practice session on each day began with a pre-practice session. During the pre-practice session, participants were provided with clear instructions regarding goals of the practice session and were motivated to achieve those goals. Specifically, the participants were instructed to match their output to the target phrase as accurately as possible, so that they are able to perform well during the retention phase. The experiment was conducted in a room free from auditory and visual distractions, and with adequate illumination. The participants were seated on a chair in front of a computer monitor prior to the start of each practice session. During practice, production of each practice trial by the participants was preceded by the orthographic and auditory representations of the target phrase. The auditory model was presented via loudspeakers. It was pre-recorded by a young adult female speaker of standard US English dialect. The orthographic model of the target phrase was presented via a PowerPoint format along with the auditory model. The initiation of the PowerPoint presentation signaled the beginning of the practice regimen. With initiation of each PowerPoint slide, the participants were able to see and hear the orthographic and auditory representations of the target phrase, respectively. The production of the speech phrase following the orthographic and auditory representations constituted one practice trial. After each production trial, the researcher pressed the 'return' key allowing the initiation of the next production trial. This was carried out until the completion of the entire 50 practice trials on each of the 2-day practice period. The participant utterances were recorded using a condenser desktop microphone placed at a distance of $12 \mathrm{~cm}$ from the participant's mouth. The output acoustic signal from the microphone was fed into a desktop computer (Dell Optiplex GX620) running Audacity 2.0.3 for acoustic analysis. 
The nature of practice differed between the constant and variable practice conditions. Participants in the constant practice condition were involved in repeated practice of the target complex phrase alone. At the end of every 10 practice trials, participants were provided with a cumulative feedback of their performance during the preceding 10 trials. Thus, at the end of 50 practice trials, there were 5 instances of feedback provided to the participants. The participants in variable practice condition practiced the target complex phrase along with an alternate complex phrase, in an attempt to satisfy the requirements of the variable practice. The alternate phrase was similar to the target phrase in terms of the phonemic composition. However, it differed from the target phrase in terms of intonation contour. The target phrase was relatively flat with no evident stress, but the alternate phrase had a rising intonation pattern at the end of the phrase. Choosing an alternate phrase that shared the same phonemic composition as the target phrase, but differed in terms of intonation satisfied the criteria of variable practice. The participants practiced 25 trials of the alternate complex phrase and another 25 trials of the target complex phrase during each day of the 2-day practice period. The target and alternate phrases were randomized across the entire 100 practice trials. At the end of every 10th trial, the participants received feedback similar to constant practice. The practice trials were randomized in a manner such that every 10th practice trial constituted the target phrase. This arrangement was made so that the participants will receive feedback on the target complex phrase alone. The above training protocol has proved beneficial for participants to learn novel utterances in a previous experiment [14].

Practice Design for the Simple Group

The simple group participants practiced a simple meaningless target phrase 'Thak willy veen Wilbur'. The simple target phrase also followed the phonotactic constraints of the English language. The simple target phrase differed from the complex target phrase in terms of the number of words and syllables. The simple target phrase had 4 non-words, 2 being bisyllabic and the other 2 words being monosyllabic. The nature of practice and feedback provision was similar to the complex group. The participants in the variable practice condition practiced the simple target phrase along with an alternate simple phrase. Similar to the complex practice condition, the alternate simple phrase differed from the target in terms of the intonation pattern. The alternate simple phrase had a rising pattern towards the ends, whereas the target simple phrase was relatively flat in terms of intonation contour. Student researchers who were trained in this procedure carried out the entire experiment.

\section{Data Collection Points}

Acquisition Phase

At the end of each day of the acquisition phase, there was break of $10 \mathrm{~min}$. After which, the participants were encouraged to produce 10 trials of the speech phrase without further practice or feedback. The participant outputs were recorded for later acoustic analyses. The data collected during first and second day of the acquisition phase served as the first and second data collection points, respectively.

\section{Retention Phase}

Participants returned on the third day for the retention phase. The participants were encouraged to produce 10 trials of the speech phrase they had practiced during the first 2 days. The par- ticipants were not allowed to practice further and did not receive feedback during the retention test. The participant outputs were recorded for later acoustic analyses. The data collected during the retention phase served as the third data collection point.

\section{Data Analysis}

The analysis focused on evaluating the production accuracy of the participants' production during the post-acquisition and retention phases by calculating the 'percentage of phonemes correct' (PPC) [21]. The rationale for including PPC is that it has been proven to be an effective outcome measure in studies investigating non-word repetition [22]. Typically, PPC is calculated by dividing the number of correct phonemes produced in an utterance by the total number of phonemes produced in the utterance and multiplying by 100 . A mean PPC value was obtained from the 10 trials during each of the post-acquisition and retention phases across all the 40 participants.

\section{Statistical Analysis}

The statistical analysis compared the performance of the participants during the acquisition and retention phases as a function of task complexity and practice variability. The mean PPC values obtained from each of the 40 participants were subjected to a 3-way mixed-model analysis of variance (ANOVA) (3 data collection points, 2 complexity levels, 2 practice conditions). The withingroup factor was the data collection point and the between-group factors were task complexity and practice condition. Significant interactions were probed using simple effects analysis with Bonferroni correction for multiple comparisons. Student researchers who were blinded to the participants' information analyzed the data.

\section{Results}

The 3-way mixed-model ANOVA revealed a significant effect of data collection point, $\mathrm{F}(2,72)=5.7, \mathrm{p}<$ 0.01 . There was also a significant effect for the task complexity, $\mathrm{F}(1,36)=9.9, \mathrm{p}<0.01$, with the PPC scores for the simple task $(M=98.66, \mathrm{SD} 5.9)$ being significantly higher than the PPC scores for the complex task $(\mathrm{M}=76$, SD 29.95). There was no major effect on the practice variability. The major effect was qualified by a significant interaction of data collection point $\times$ task complexity, $\mathrm{F}(2,72)=5.6, \mathrm{p}<0.01$. All other interactions were insignificant.

The simple effect analysis that probed the interactive effect of data collection point $\times$ task complexity, revealed that the PPC scores of participants practicing the simple phrase was significantly higher than those practicing the complex phrase on first $(\mathrm{p}<0.01)$ and third data collection points $(\mathrm{p}<0.01)$. Conversely, the participants who practiced the complex phrase had significantly higher PPC scores during the second data collection point in comparison to the first $(\mathrm{p}<0.01)$ and third data collection 
Table 1. Mean percentage of phoneme correct scores (\%) of participants who practiced simple and complex phrases in constant and variable practice conditions across the 3 data collection points

\begin{tabular}{|c|c|c|c|c|c|c|c|c|c|c|c|}
\hline \multicolumn{6}{|l|}{ Simple } & \multicolumn{6}{|l|}{ Complex } \\
\hline Day 1 & Day 2 & Day 3 & Day 1 & Day 2 & Day 3 & Day 1 & Day 2 & Day 3 & Day 1 & Day 2 & Day 3 \\
\hline $96.41(8.1)$ & $96.41(8.1)$ & $97.62(6.8)$ & $99.33(2.1)$ & $100(0)$ & $100(0)$ & $81.61(30.5)$ & $99.61(1.2)$ & $85.23(29.8)$ & $70.39(29.9)$ & $98.92(2.4)$ & $82.76(28.2)$ \\
\hline
\end{tabular}

The SD values are indicated in parentheses.

points ( $\mathrm{p}<0.01)$. The PPC scores of the participants who practiced simple and complex phrases in constant and variable practice conditions are shown in table 1.

\section{Measurement Reliability}

Inter-rater measurement reliability for the calculation of the outcome measure (PPC) was based on randomly choosing and re-measuring $20 \%$ of the data (i.e., 8 of 40 participants) and performing a Pearson correlation. The inter-rater reliability of the original and re-measured data was $r=0.99(p<0.05)$.

\section{Discussion}

This is the first study to systematically investigate the interactive effect of task complexity and practice variability on speech-motor learning. The results suggest that there is no difference between constant and variable practice on speech-motor learning, irrespective of the complexity of the task that was learned. In other words, there is no interaction between task complexity and practice variability.

Interestingly, the results of this study revealed no significant effect of practice variability. Adams and Page [8] compared constant versus variable practice in 40 healthy participants. One group of participants practiced 50 trials of the utterance 'Buy Bobby a Poppy' with the target duration of $2.4 \mathrm{~s}$ (constant practice), and the other group practiced 50 trials of the same utterance with the target durations of 2.4 and $3.6 \mathrm{~s}$ (variable practice). The participants underwent a retention test 2 days after the acquisition phase and produced the target utterance without further practice. The outcome measure was absolute error (AE), which was determined by calculating the absolute difference between the target utterance duration and the participants' utterance durations. The AE score was obtained for the $2.4 \mathrm{~s}$ target duration. The results revealed that both

Interaction of Task Complexity and

Practice in Speech-Motor Learning groups demonstrated similar performance during the acquisition phase, but the retention test revealed the variable practice group to have significantly lower $\mathrm{AE}$ than the constant practice group. The findings of this study are in disagreement with Adams and Page's study. The main reason for this discrepancy could be attributed to the nature of the to-be-learned task. The participants in the Adams and Page's study were involved in a temporal learning task. Whereas participants in this study were involved in a spatial learning task, which refers to learning the movement associated with each articulatory gesture in a word or phrase. In spatial learning, the focus is more on the accuracy with which the motor movements are executed rather than the timing of movements. It is possible that the effects of practice variability is observed in temporal learning tasks, which involves learning the timing of movements rather than spatial learning tasks. So it is not surprising that the effect of practice variability was not observed in this study that required participants to learn a spatial task as opposed to a temporal task.

In terms of interaction between task complexity and data collection points, findings revealed that participants who practiced the simple phrase on days 1 and 3 had better PPC scores than participants who practiced the complex phrase. The main reason for this finding is that participants involved in practicing the simple phrase reached a ceiling effect from day 1 , thereby leaving no room for any improvement in their performance during the other 2 days. The ceiling effect was evident from the PPC scores obtained by participants who practiced the simple phrase, irrespective of the type of practice. On the contrary, participants involved in practicing the complex phrase required an entire set of 50 trials to demonstrate an improvement in their performance, as revealed by their PPC scores on day 2 . However, as these participants abstained from further practice of the complex phrase after day 2 , it is possible that it could have resulted in the decay of memory trace that was associated with complex phrase. 


\section{Conclusion}

This study is not without limitations. A low sample size ( $\mathrm{n}=40$ ) could affect the generalization of the current findings. It is important to note that the current findings were based on training the participants for just 2 consecutive days. It is possible that extended period of practice could have influenced the learning outcome. In spite of these limitations, this study suggests that the effect of practice variability on speech-motor learning is not influenced by the complexity of the to-be learned task. Another important implication from this study is that using a simple task can lead to a ceiling effect, so researchers and clinicians should be cautious in selecting appropriate speech stimulus/stimuli for practice. The findings from this study can have major ramifications in speech therapy, where often there is interaction between the speech task and practice conditions.

\section{Acknowledgements}

The author would like to thank Roha Mariam Thomas for her ideas and all the student researchers for their assistance in collecting and analyzing the data.

\section{Authorship Contributions}

R.K. designed the study, analyzed the data, performed statistical analysis and wrote the manuscript.

\section{Disclosure Statement}

The author has no financial or non-financial conflict of interest to report. This paper complies with International Committee of Medical Journal Editor's standards.

\section{References}

1 Schmidt RA, Lee T: Motor Control and Learning: A Behavioral Emphasis, ed 5. Champaign, Human Kinetics, 2011.

2 Austermann Hula SN, Robin DA, Maas E, Ballard KJ, Schmidt RA: Effects of feedback frequency and timing on acquisition, retention, and transfer of speech skills in acquired apraxia of speech. J Speech Lang Hear Res 2008;51:1088-1113.

3 Friedman I, Hancock AB, Schulz G, Bamdad $\mathrm{MJ}$ : Using principles of motor learning to treat apraxia of speech after traumatic brain injury. J Med Speech Lang Pathol 2010;18:13-34.

4 Maas E, Robin DA, Austermann Hula SN, Freedman SE, Wulf G, Ballard KJ, et al: Principles of motor learning in treatment of motor speech disorders. Am J Speech Lang Pathol 2008; 17:277-298.

5 Poole JL: Application of motor learning principles in occupational therapy. Am J Occup Ther 1991;45:531-537.

6 Bislick LP, Weir PC, Spencer K, Kendall D, Yorkston KM: Do principles of motor learning enhance retention and transfer of speech skills? A systematic review. Aphasiology 2012; 26:709-728.

7 Kawashima R, Tajima N, Yoshida H, Okita K, Sasaki T, Schormann T, et al: The effect of verbal feedback on motor learning - a PET study. Positron emission tomography. Neuroimage 2000;12:698-706
8 Adams SG, Page AD: Effects of selected practice and feedback variables on speech motor learning. J Med Speech Lang Pathol 2000;8: 215-220.

9 Knock TR, Ballard KJ, Robin DA, Schmidt RA: Influence of order of stimulus presentation on speech motor learning: a principled approach to treatment for apraxia of speech. Aphasiology 2000;14:653-668.

10 Adams SG, Page AD, Jog M: Summary feedback schedules and speech motor learning in Parkinson's disease. J Med Speech Lang Pathol 2002;10:215-220.

11 Steinhauer K, Grayhack JP: The role of knowledge of results in performance and learning of a voice motor task. J Voice 2000;14:137-145.

12 Maas E, Farinella KA: Random versus blocked practice in treatment for childhood apraxia of speech. J Speech Lang Hear Res 2012;55:561578.

13 Wong AW, Whitehill TL, Ma EP, Masters R: Effects of practice schedules on speech motor learning. Int J Speech Lang Pathol 2013;15: 511-523.

14 Kaipa R: Evaluation of Principles of Motor Learning in Speech and Non-Speech-Motor Learning Tasks. Christchurch, University of Canterbury, 2013.

15 Ballard KJ, Maas E, Robin DA: Treating control of voicing in apraxia of speech with variable practice. Aphasiology 2007;21:11951217.
16 Wong AY, Ma EP, Yiu EM: Effects of practice variability on learning of relaxed phonation in vocally hyperfunctional speakers. J Voice 2011;25:e103-e113.

17 Wulf G, Shea CH: Understanding the role of augmented feedback: the good, the bad, and the ugly; in Williams AM, Hodges NJ (eds): Skill Acquisition in Sport: Research, Theory and Practice. London, Routledge, 2004, pp 121-144.

18 Wambaugh JL, Nessler C, Cameron R, Mauszycki SC: Treatment for acquired apraxia of speech: examination of treatment intensity and practice schedule. Am J Speech Lang Pathol 2013;22:84-102.

19 Kaipa R, Jones RD, Robb MP: Are individuals with Parkinson's disease capable of speechmotor learning? - a preliminary evaluation. Parkinsonism Relat Disord 2016;28:141145.

20 Shea CH, Kohl RM: Composition of practice: influence on the retention of motor skills. Res Q Exerc Sport 1991;62:187-195.

21 Shriberg LD, Austin D, Lewis BA, McSweeny JL, Wilson DL: The percentage of consonants correct (PCC) metric: extensions and reliability data. J Speech Lang Hear Res 1997;40:708722

22 Dollaghan C, Campbell TF: Nonword repetition and child language impairment. J Speech Lang Hear Res 1998;41:1136-1146. 